\title{
Processing of Alternated and Inverse Fonts in Word Recognition by Native and Nonnative Speakers of English
}

\author{
By Hye K. Pae \\ Yong-Won Lee
}

\begin{abstract}
This study investigated the perceptibility of visually altered stimuli in word recognition by English- and Korean-speaking adults. Forty four college students from the U.S. and South Korea participated in this study. The orthographic depth hypothesis and the psycholinguistic grain size theory guided this study. Three different fonts (i.e., normal, alternated, and inverse fonts) and two lexical features (i.e., words and nonwords) were used in a lexical decision task. The results showed that native English speakers were less sensitive to the visually noisy shapes (i.e., alternated and inverse fonts) than the Korean participants. It suggests that the psycholinguistic unit of the first language plays a role in word recognition beyond the language dominance (i.e., first language vs. foreign language).
\end{abstract}

\section{Introduction}

Word identification has long drawn researchers' attention, as word recognition is the foundation of reading processes and serves as a precursor to reading success (Adams, 1990). With the contributions of previous studies, the extant body of knowledge has been broadened and deepened with respect to the interaction among componential processes in reading. However, what still remains unclear is how lexical access is characterized by visual shapes and the extent to which lexical processing is similar or different across speakers of different first languages (L1s). One way to tackle this inquiry is to examine lexical processing without phonological and semantic manipulations in instruments with two groups of speakers whose L1s share the same alphabetic principle but have different scripts. English and Korean meet this condition well. This study examined how native Korean speakers resolve visually noisy stimuli in English as a foreign language (FL), in comparison to native English speakers. Since the two groups spoke alphabetic languages as L1s, the rationale for this study is three-fold in terms of the linguistic properties of their L1s: (1) commonalities shared between English and Korean as alphabetic languages, (2) differences in visual scripts (horizontal linear arrangement vs. square-shaped

${ }^{*}$ Associate Professor, University of Cincinnati, USA.

${ }^{\dagger}$ Associate Professor, Seoul National University, South Korea. 
block; e.g., school vs. 학교), and (3) intraword properties (alphabetic vs. alphasyllabary) characterized by the role of vowels. The orthographic depth hypothesis and psycholinguistic grain size theory served as theoretical frameworks.

\section{Literature Review}

\section{Orthographic Depth and Word Recognition}

One of the goals of research into visual word recognition is to identify the features of lexical structures that influence visual processing and phonological mediation. The orthographic depth hypothesis (Frost, 1994; Frost, Katz, \& Bentin, 1987), which uses the regularity of a grapheme-to-phoneme conversion (GPC) as a criterion for a shallow or deep orthography, offers a fine foundation to understand the mechanism of this interaction. Shallow orthographies have a regular and consistent mapping between spelling and sound, while deep orthographies have an irregular and unpredictable association between spelling and sound. The orthographic depth hypothesis posits that phonological information is activated to recognize words before lexical access in orthographically shallow languages (e.g., Spanish, Italian, Korean), whereas it is activated after lexical access in orthographically deep languages (e.g., English, Hebrew; Frost, 1994).

In connection with the orthographic depth hypothesis, the dual-route and connectionist models have been leading theoretical frameworks in the word recognition realm. The dual-route cascaded model posits two paths (i.e., lexical and sublexical routes) for translating print into sound (Rastle \& Coltheart, 1999). Of the two paths, one is the lexical route (a.k.a., addressed, worddetector, or lexical look-up route), which involves visual, automatic, and direct access to stored orthographic and phonological representations. The direct lexical route makes word recognition an automatic top-down processing. Deep orthographies are processed via the lexical route for irregular words. It is especially useful for the correct pronunciation of irregular words that violate the conventional GPC rule. The other route is the sublexical route (a.k.a., nonlexical, assembly, rule-based, or GPC procedure), which utilizes a rulegoverned (i.e., GPC) process that maps the constituent graphemes onto corresponding phonemes. Shallow orthographies are typically accessed via the GPC route (Frost, 1994). Although English is characterized as a deep orthography, regular words are processed via the GPC route as well due to the consistent mapping from the letter to the sound. The GPC route makes visual processing slower than direct lexical route for high frequency words due to an additional conversion from orthography to phonology (Stone \& Van Orden, 1993). As far as low frequency words are concerned, however, the processing speed is reversed; that is, the lexical route takes longer because of weak representations of words stored in the mental lexicon. The GPC route processing is not susceptible to word frequency due to the consistent spellingsound mapping. 
The other line of theoretical view in the word recognition area is the connectionist parallel distributed processing (PDP) model (Plaut, McClelland, Seidenberg, et al., 1996; Seidenberg \& McClelland, 1989). The PDP model does not differentiate a lexicon from a GPC assembly process, but involves a pattern of connected activation of processing units in terms of the orthographic, phonological, and semantic characteristics of a letter string. According to the PDP model, both words and nonwords are not represented locally as wordspecific units, but are represented at an elementary level of associative networks in a connected fashion. The dual-route and PDP models differ in terms of presence (the dual-route model) or lack (the PDP model) of lexicons, and provide different explanations about the nature and locus of word frequency and nonword reading process.

\section{Psycholinguistic Grain Size Theory}

The efficiency of mapping from orthographic elements to phonological equivalents varies across languages because of the different granularity of the writing system (Ziegler \& Goswami, 2005). According to the psycholinguistic grain size theory (Ziegler \& Goswami, 2005), readers of shallow orthographies make use of small grain sizes (e.g., graphemes) due to the consistent GPC. Readers of deep orthographies rely on larger grain sizes (e.g., onsets, rimes), because a reliance on the small grain size only leads to errors resulting from mismatches between letters and sounds. Hence, English readers need to develop both small-unit and large-unit sublexical recoding strategies in parallel. This is consistent with the flexible-unit-size hypothesis (Brown \& Deavers, 1999; Ziegler \& Goswami, 2005) in English word identification.

\section{L1 Influence on L2 English Word Recognition}

Word recognition has been expanded to various scripts in the recent three decades. The orthographic feature of each language has been considered to play a role in word reading with respect to the orthographic depth, psycholinguistic grain size, and minimal processing unit (Ziegler \& Goswami, 2005). Specifically, the minimal unit of alphabetic languages, such as English, Spanish, and French, is a phoneme, whereas that of logographic languages, such as Chinese and Japanese, is a syllable. Korean is viewed as an alphabetic language in that each grapheme groups together to form a syllabic unit. Unlike the Roman alphabetic languages, however, it has unique characteristics of syllabic dominance (i.e., graphemes are not arranged in a horizontally linear form but grouped together in a square-like block with a vowel at the core; 하교 $\rightarrow$ 학교); hence, it is characterized as alphabetic syllabary or alphasyllabary (Pae, 2011).

At the center of recent studies in second language acquisition are different linguistic or writing systems, as many studies have been conducted in crosslanguage comparisons. A number of research studies have investigated different writing systems in the spectrum of phonological, orthographic, and syntactic structures as well as word recognition and sentence processing (Akamatsu, 1999, 2003; Chikamatsu, 2006). Akamatsu (1999, 2003) 
investigated the effect of the L1 orthographic feature on word recognition in English as a second language (L2). Using a mixture of normal fonts and visually distorted fonts, Akamatsu (1999, 2003) compared Iranians, Chinese, and Japanese students' reading performance with native English speakers. The results showed that Iranian students whose L1 was alphabetic were less affected by the case alternation than Chinese and Japanese counterparts whose L1s were syllabic, suggesting that L1 orthographic features influenced L2 English reading.

\section{The Present Study}

Despite a wealth of studies that examined different modes of word recognition in various linguistic systems, there is a paucity in research into how visual noise is resolved especially in inverse fonts. Given that Korean and English share commonalities in the alphabetic principle, but have two distinctly different scripts (book vs. 책), the purpose of this study was to examine the performance pattern of native English speakers and Korean speakers in word recognition. Three stimuli shapes were utilized in a lexical decision task for this study: Regular fonts, alternated fonts, and upside-down fonts. The last two fonts had visual noise with reduced word-shape cues. Two research questions guided this study.

1. How do native English speakers and Korean speakers who learned English as a foreign language $(\mathrm{FL})^{1}$ process visually altered stimuli?

2. How is their performance (i.e., accuracy and latency) on the three different shapes (i.e., normal, alternated, and upside-down fonts) different from each other?

The first question was posed because of the different orthographic depth in the participants' L1s. Given the language dominance (i.e., L1 vs. FL), hypothesized was that there would be differences in the performance of the two groups, but the difference would be modest because the two groups of participants had the same alphabetic language as L1s. The second question was posed in order to determine whether or not the difference in the grain size (i.e., onsets-rimes vs. graphemes) of the participant's L1 played a role in resolving visually distorted stimuli. Hypothesized was that the participants would have a different resolution pattern of visual noises, if not great, because their L1s' intraword units were different (large grain size vs. small grain size).

\footnotetext{
${ }^{1}$ Although the Korean participants learned English as a foreign language (FL), FL and L2 are used interchangeably in this paper for the sake of consistency with the literature and convenience.
} 


\section{Method}

\section{Participants}

A total of forty-four college students from two international sites participated in this study. The first group was composed of 18 Englishspeaking undergraduate and graduate students at the University of Cincinnati in the U.S (mean age $=28.12, \mathrm{SD}=5.83$, range $=21-38$; female: $67 \% ; 14$ Caucasians, 3 African Americans, and 1 mixed), while the second group comprised 26 English majors who learned English as FL at one of National Universities in South Korea (mean age $=21.65, \mathrm{SD}=2.65$, range $=19-30$; female: $85 \%$ ). The Korean students' English proficiency level was high, as all of them were English majors. The mean score of the TOEFL iBT test of the participants who self-reported was 110.61. Therefore, it was clear that their performance on word recognition was not disadvantaged in comparison to that of native speakers, which granted fair comparability between the two groups.

\section{Procedure}

A computerized test was individually administered in a quiet room. Each participant signed on a written consent form prior to the test and filled out a demographic questionnaire afterward. The stimulus was presented visually one at a time and was randomized upon presentation. The presentation of the stimuli on the computer screen began with a fixation point $(+)$ for 500 milliseconds (ms), followed by 6 practice items and 60 target stimuli.

\section{Instrument}

A lexical decision task was constructed for this study, using the DMDX ${ }^{1}$ program. Sixty stimuli were drawn from the Word Identification subtest of the Woodcock Reading Mastery Test-NU (WRMT; Woodcock, 1998). The splithalf reliability coefficient for the college normative sample was .94 and that for adults was .97 (Woodcock, 1998). The 60 words were systematically organized to have three different visual displays, including normal fonts, alternated fonts, and upside-down fonts as well as two different letter string types, including words and nonwords.

\section{Data Analysis}

Descriptive statistics, an analysis of variance (ANOVA), and $t$-tests were employed to examine the research questions. The results of a correlation matrix and hierarchical regression analyses are not reported in this paper.

\footnotetext{
${ }^{1}$ The DMDX is a freely available online: http://www.u.arizona.edu/ kforster/dmdx/downlo ad.htm. Credit should be given to Kenneth Foster and his colleagues at the University of Arizona.
} 


\section{Results}

Table 1 shows the descriptive statistics of the variables. The largest mean difference in the accuracy rate among the three visual shapes was observed in the upside-down shapes, followed by the alternated fonts. The native speakers were less influenced by the distorted shapes than Korean participants (mean difference: 4.75 vs. 5.77 for the alternated fonts, 8.88 vs. 11.14 for the upsidedown fonts).

Table 1. Descriptive Statistics

\begin{tabular}{|c|c|c|c|c|c|c|c|c|}
\hline & \multicolumn{2}{|c|}{ Overall } & \multicolumn{2}{|c|}{$\underline{\text { Normal Fonts }}$} & \multicolumn{2}{|c|}{$\underline{\text { Alternated Fonts }}$} & \multicolumn{2}{|c|}{ Upside-Down Fonts } \\
\hline & Accuracy & Latency & Accuracy & Latency & Accuracy & Latency & Accuracy & Latency \\
\hline Koreans & $\begin{array}{c}83.78 \\
(17.69) \\
\end{array}$ & $\begin{array}{l}1433.79 \\
(515.67) \\
\end{array}$ & $\begin{array}{c}89.42 \\
(19.37) \\
\end{array}$ & $\begin{array}{l}1004.02 \\
(200.81) \\
\end{array}$ & $\begin{array}{c}83.65 \\
(14.11) \\
\end{array}$ & $\begin{array}{l}1336.47 \\
(274.01) \\
\end{array}$ & $\begin{array}{c}78.27 \\
(18.22) \\
\end{array}$ & $\begin{array}{l}1960.88 \\
(462.04) \\
\end{array}$ \\
\hline $\begin{array}{l}\text { Native } \\
\text { Speakers }\end{array}$ & $\begin{array}{c}90.83 \\
(12.93)\end{array}$ & $\begin{array}{l}1477.03 \\
(503.30)\end{array}$ & $\begin{array}{l}95.55 \\
(7.34)\end{array}$ & $\begin{array}{l}1090.09 \\
(179.10)\end{array}$ & $\begin{array}{c}90.28 \\
(14.52)\end{array}$ & $\begin{array}{l}1378.08 \\
(291.01)\end{array}$ & $\begin{array}{c}86.67 \\
(14.58)\end{array}$ & $\begin{array}{l}1962.93 \\
(503.40)\end{array}$ \\
\hline $\begin{array}{c}\text { Mean } \\
\text { difference }\end{array}$ & 7.05 & 43.24 & 6.13 & 86.07 & 6.63 & 41.61 & 8.4 & 2.05 \\
\hline
\end{tabular}

Note: Standard Deviations in Parentheses; latency indicates millisecond; accuracy represents percentages correct

$* \mathrm{p}<.05, * *<\mathrm{p}<.01, * * * \mathrm{p}<.001$

RQ 1: How do Native English Speakers and Korean Speakers who Learned English as a Foreign Language $(F L)^{l}$ Process Visually altered Stimuli?

The two groups' performance on the lexical decision test was compared. A $2 \times 3$ analysis of variance (ANOVA) was performed with the two L1 groups as a between-subject factor and the three visual shapes as a within-subject variable. For accuracy, there was a significant main effect of the L1 on the performance of the two groups, $F(1,42)=17.10, p<.001, \eta_{p}{ }^{2}=.289$. A main effect of the three visual shapes was also found: $F(2,42)=5.57, \mathrm{p}<.05, \eta_{\mathrm{p}}{ }^{2}=.117$. There was a nonsignificant interaction effect between the two L1s and the three visual shapes, indicating that the performance on the three visual shapes was not affected differently by L1s. Concerning latency, a two-way ANOVA showed no main effect with the L1 $(p>.05)$. There was a significant main effect among the visual shapes $\left(\mathrm{F}(2,42)=113.85, p<.001, \eta_{\mathrm{p}}{ }^{2}=.731\right)$. Tukey HSD post-hoc test revealed that all the three combinations were significantly different $(p s<.001)$. No interaction effect was found between the different L1s and different visual shapes, meaning that the participant's L1 did not affect the performance on the visual shapes.

With regard to item analyses, a 2 × 3 ANOVA was performed with two L1 groups as a between-subject variable and three shapes as a within-subject factor. The result of Levene's test of equality of error variances was nonsignificant $(p>.05)$, suggesting the comparability of the two groups. First, a two-way ANOVA was performed with the accuracy data to determine if there

\footnotetext{
${ }^{1}$ Although the Korean participants learned English as a foreign language (FL), FL and L2 are used interchangeably in this paper for the sake of consistency with the literature and convenience.
} 
was interaction between different L1s and three shapes by comparing how the performance was affected by the L1s and by the visual shapes. There was a significant difference in the main effect between two groups $(\mathrm{F}(1,114)=6.47$, $\left.\mathrm{p}<.05, \eta_{\mathrm{p}}^{2}=.05\right)$. The mean of the native speakers was significantly greater than that of Korean learners of English. There was a significant main effect among three visual shapes $\left(\mathrm{F}(2,114)=4.37, p<.05, \eta_{\mathrm{p}}^{2}=.07\right)$. Tukey HSD post-hoc test showed that the mean of normal fonts was significantly greater than that of upside-down fonts $(p<.05)$, but there was no significant difference in shapes between normal fonts and alternated fonts or between alternated fonts and upside-down shapes. However, no interaction effect was found. Next, a two-way ANOVA was performed with the latency data. A main effect was found only with the visual shape $\left(\mathrm{F}(2,114)=74.31, p<.001, \eta_{\mathrm{p}}{ }^{2}=.566\right)$. There was no significant main effect between the two L1 groups. No interaction effect was found between the different L1s and different visual shapes, meaning that the effect of the visual shapes did not differ depending on the participants' L1 status. With respect to accuracy, there was no significant difference found in the three visual shapes for both native speakers and Korean English learners. As for the latency, there were significant differences for both groups $\left(\mathrm{F}(2,57)=32.06, p<.001, \eta_{\mathrm{p}}{ }^{2}=.529\right.$ for the native speakers; $\mathrm{F}(2,57)$ $=43.05, p=.001, \eta_{\mathrm{p}}{ }^{2}=.602$ for the Korean participants).

Since previous research has documented that the efficiency of word recognition is closely related to frequent words, word frequency was also examined. The mean frequency of the base words was 134781, ranging from 16 to 2912708 per million words. The mean number of letters of the base words was 6.43, ranging from 4 to 11 letters. The number of syllables ranged from 1 to 5. The two groups performed differently in accuracy and latency, according to word frequency: For the Korean participants, $\mathrm{F}(1,58)=5.28, p<$ .05 for accuracy, $\mathrm{F}(1,58)=5.25, p<.05$ for latency; For the native English speakers, $\mathrm{F}(1,58)=16.38, p<.001$ for accuracy, $\mathrm{F}(1,58)=8.84, p<.001$ for latency.

When word frequency was controlled, a one-way ANOVA did not produce a significant difference in accuracy by the three visual shapes. However, a oneway ANOVA revealed significant differences in latency by the three shapes for the two groups, when word frequency was taken into account: For the Korean English learners, $F(2,56)=43.20, p<.001 ; \eta_{\mathrm{p}}{ }^{2}=.61$; for the native speakers, $F(2,56)=33.23, p<.001 ; \eta_{\mathrm{p}}{ }^{2}=.54$. When the number of letters was controlled, the three visual fonts produced significant differences in latency: For the Korean English learners, $F(2,56)=50.18, p=.000 ; \eta_{\mathrm{p}}{ }^{2}=.64$; for the native speakers, $F(2,56)=39.76, p<.001 ; \eta_{\mathrm{p}}^{2}=.59$.

$R Q$ 2: How is their Performance (i.e., accuracy and latency) on the Three Different Shapes (i.e., normal, alternated, and upside-down fonts) Different from Each Other?

In order to examine the extent to which the three visual shapes affected the two groups' performance on the lexical decision task, analyses were run by word frequency as well as the lexical feature (i.e., words and nonwords). The 
effect of word frequency was found in the accuracy rate of high frequency stimuli for the alternated fonts and upside-down fonts in which the native speakers of English outperformed the Korean counterparts $(t(1)=2.69, \mathrm{p}<$ $.05, t(1)=2.72, \mathrm{p}<.05$, respectively; see Figure 1). In general, words that were more frequent were processed faster than those that were less frequent. The Korean participants did not seem to be influenced by word frequency except the high frequency upside-down fonts. Interestingly, the Korean students processed the stimuli faster than the native speakers except the high frequency stimuli of the alternated and upside-down fonts. This cast suspicion of a trade-off effect. A close look into a possibility of the accuracy-speed tradeoff effect was made by directly comparing the item accuracy score against its latency and by comparing each participant's latency of each item against the mean scores of accuracy and latency. The result did not yield a systematic pattern of accuracy-speed trade-off in the Korean speakers' performance. It was possible that the Korean students took random guess for the low frequency words of the visually distorted fonts. Considering Adams (1990) assertion that proficient readers are "sophisticated guessers," this does not directly lead to the low quality of data.

Figure 1. The Performance of the Koreans and Native Speakers on the Visual Shapes by Word Frequency
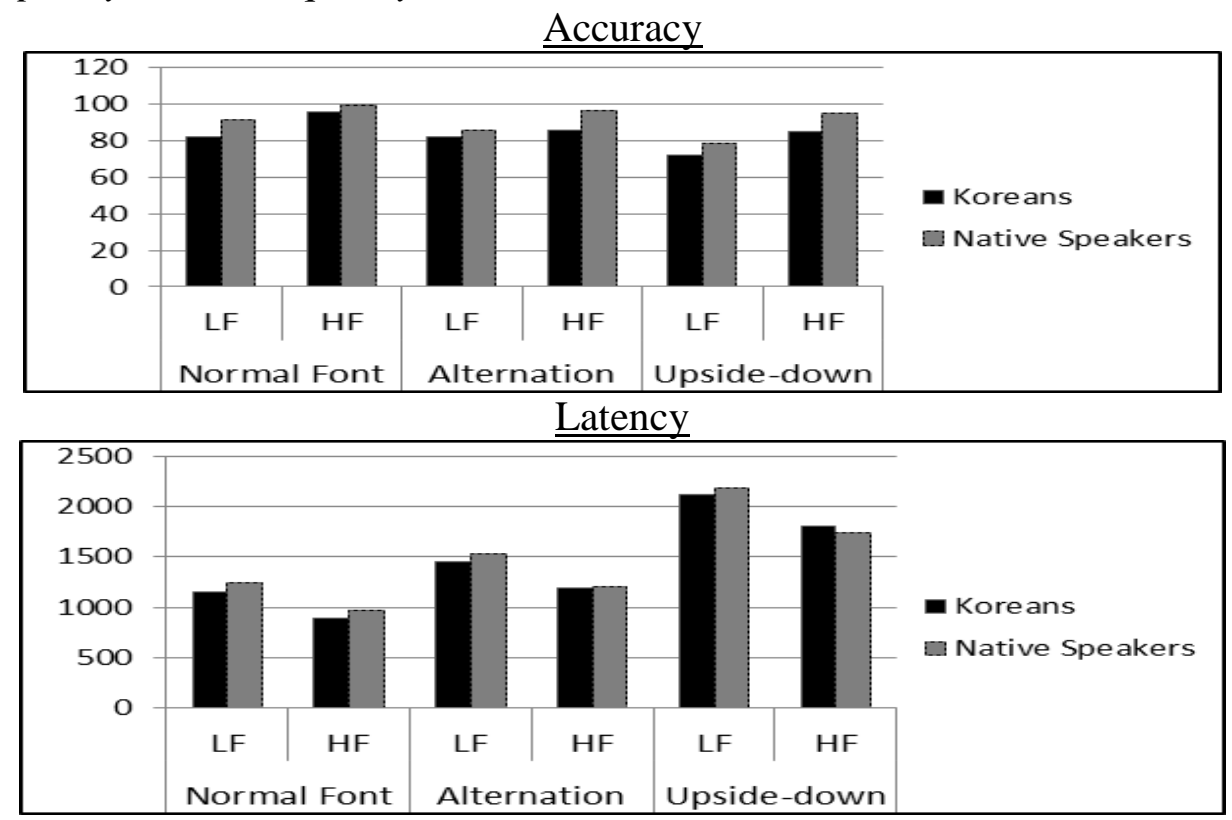

Next, the participants' performance on the lexical decision task was examined based on the lexical features (i.e., words or nonwords). Both groups show consistent performance across the word or nonword stimuli with respect to accuracy. However, the two groups showed a significantly different performance on latency across the two features. The lexical features were also examined by the three different visual shapes. The Korean participants processed the stimuli slightly faster than the native counterparts, except alternated nonword and upside-down word displays (see Figure 2). The latency 
between words and nonwords of the normal fonts was statistically significant $(t(1)=2.69, \mathrm{p}<.05)$. Again, trade-off effects were suspected, but a concordance analysis between accuracy and latency showed no systematic compensatory effects.

Figure 2. The Latency of the Koreans and Native Speakers on the Visual Shapes by Lexical Features

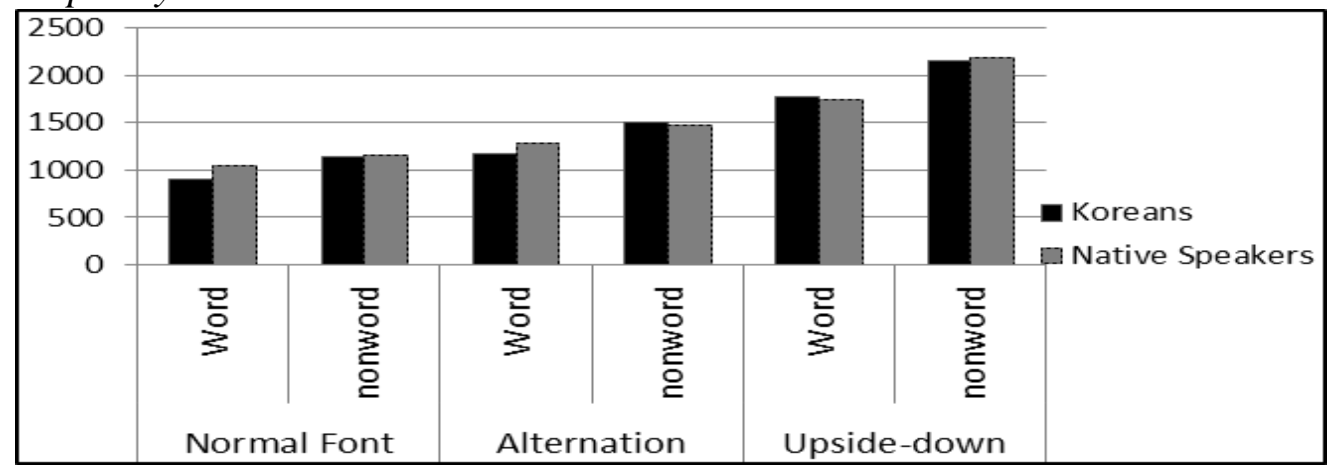

\section{Discussion}

The results of this study showed that the difference in L1s was not affected by the visual shapes of the stimuli. It seemed that the same alphabetic principle shared by the two L1s played a role in visual processing. The native speakers outperformed the Korean counterparts in accuracy. It was not surprising given the L1 and FL status for the two groups. However, the Korean participants were faster than the native counterparts in processing the visual stimuli regardless of the lexical feature (i.e., words or nonwords) and frequency (i.e., high or low frequency), except for the high-frequency upside-down fonts. This could be explained through the L1 effect on L2 word processing (Akamatsu, 1999, 2003).

The hypothesis of the lexical route applies to high-frequency words, not necessarily to low-frequency ones. Besides, English as a deep orthography requires a mixture of small- and large-unit strategies in word decoding (Ziegler \& Goswami, 2005). The bigger grain size in English than Korean might have forced the native English speakers to use the flexible psycholinguistic unit size (i.e., small and large grain sizes). If the utility of flexible unit sizes was applicable to English native speakers only, Korean learners of English as L2 or FL would not show a systematic pattern that was congruent with that of the native speakers. The magnitude of difference between high and low frequency words for the Korean was smaller than the native participants. The Korean participants' faster processing speed might have resulted from the nature of the instrument used in the study, which was a lexical decision test. For Korean L2 readers, phonology might not be mandatory to judge whether the stimulus string was a real word or not in lexical decision tests, whereas it is required in naming tests. The weaker frequency effect displayed by the Koreans could be viewed as L1 effects. This line of speculation was made in a comparison study 
of Chinese-, Japanese-, and Persian-speaking adults reading English as L2, that showed robust effects of L1 orthographic features on word-recognition processing in English as L2 (Akamatsu, 1999, 2003). From this perspective, the L1 linguistic system seemed to serve as a template for processing L2. Another explanation would be weak support for the dual-route model in L2 word recognition. It could be postulated that all lexical knowledge required for the processing of the three visual shapes might have been represented in the associated networks, as hypothesized in the PDP model (Siedenberg \& McClelland, 1989). It is likely that psycholinguistic grain sizes utilized for high- and low-frequency word recognition are interconnected and accessed in a similar manner.

Theoretical inferences drawn from the results of this study led to a conclusion that similarities and dissimilarities of alphabetic word processing were demonstrated in the speakers of similar languages of one with a pure alphabet and the other with an alphasyllabary. The commonality lied in the capacity of efficiently distinguishing words from nonwords. Since the word was recognized holistically, it was speculated that skillful readers developed "engrams or templates - pattern recognizers, as it were - for familiar words as wholes" (Adams, 1990, p. 96). It appeared that skilled readers processed visual shape information rapidly, and speakers of FL could be as efficient as native speakers in English word identification once automaticity had been acquired. This might be plausible because English has phonotactic constraints that help readers predict the letter sequence of words and because skillful readers are equipped with automaticity in recognizing whole words. Since the phonotactic constraints characterize the likelihood of grapheme combinations in English, expert readers take advantage of such sequence to accelerate visual processing. As a result, proficient readers could process a letter string with remarkable facility and speed to make use of their prediction to guide their visual inspection of words.

The difference between the two groups lied in the strength of relationships between accuracy and speed, word frequency effects, and processing speed of diverse visual shapes. When they processed letter strings in different forms of displays, Koreans were more influenced than native English speakers by the distorted visual shapes which had reduced word-cue values (i.e., nonwords). These differences seemed to stem from the fundamental difference in L1 word processing, as the dual-route processing model suggests. The results were also in line with previous research into cross-language transfer from L1 to L2 in reading. The findings of this study indicated that, although individuals who had similar L1 backgrounds (in this case, alphabetic L1s) processed English words similarly beyond the L1 and L2 status, the specificity of L1 affected English word recognition. Consistent with the findings of Akamatsu (1999, 2003), the native speakers were less affected by the visually noisy shapes than the Korean counterpart.

To summarize, although this study did not directly test the regularity or consistency of GPC, the findings demonstrated that English words were recognized differently by different $\mathrm{L} 1$ groups. This indicated that, although the same stimulus was given, the processing mechanism was conditioned by the L1 
linguistic systems. At the same time, it also suggested that L1 and L2 processing itself might be different. Because of the absence of data that allowed for a contrast of L1 and L2 matched performance, further speculation was limited. It would be interesting to disentangle the intricacy involved in L1 and L2 word recognition by speakers of L1 that showed similarities in the core linguistic systems but drastic differences in the scripts.

This study contributes to the area of word recognition by providing empirical evidence in English word recognition by individuals whose L1s have similar intralinguistic characteristics but have drastic differences in appearance (again, English and Korean are alphabetic in nature but show radical dissimilarities in written forms). Despite the merit this study has, some limitations need to be noted in relation to future directions. First, this study did not take phonology into account because of the focal point of visual resolution. Since reading is basically translating written texts into phonological forms, further studies that address phonology would broaden the understanding of word recognition processes in cross-languages. Secondly, since the participants are adults, this study does not provide a developmental aspect of word recognition. Comparative studies of children's and adults' processing of words would provide insights into developmental trajectories. Next, since the Korean participants were highly functioning college students, it is unclear whether the results of this study can be generalized to average or lower performers in English as L2. More studies with diverse English skills are needed to corroborate the findings of this study. Lastly, an inclusion of other L1 groups would add an interesting picture to the cross-linguistic L1 influence on L2 word identification. Subsequent research is recommended to include groups that have different L1 systems, such as Japanese and Chinese.

\section{References}

Adams, M. J., 1990. Beginning to read: Thinking and learning about print. Cambridge, MA: MIT Press.

Akamatsu, N., 1999. The effects of first language orthographic features on word recognition processing in English as a second language. Reading and Writing: An Interdisciplinary Journal, 11, pp.381-403.

Akamatsu, N., 2003. The effects of first language orthographic features on second language reading in text. Language Learning, 53, pp.207-231.

Brown, G. D. A., \& Deavers, R. P., 1999. Units of analysis in nonword reading: Evidence from children and adults. Journal of Experimental Child Psychology, 73, pp.208-242.

Chikamatsu, N., 2006. Developmental word recognition: A study of L1 English readers of L2 Japanese. Modern Language Journal, 90, pp.67-85.

Frost, R., Katz, L., \& Bentin, S., 1987. Strategies for visual word recognition and orthographical depth: A multilingual comparison. Journal of Experimental Psychology: Human Perception and Performance, 13, pp.104-115.

Frost, R., 1994. Prelexical and postlexical strategies in reading: Evidence from a deep and a shallow orthography. Journal of Experimental Psychology: Learning, Memory, and Cognition, 29, pp.116-129. 
Pae, H. K., 2011. Is Korean a syllabic alphabet or an alphabetic syllabary? Writing Systems Research, 3(2), pp.103-115.

Plaut, D. C., McClelland, J. L., Siedenberg, M. S., \& Pattterson, K., 1996. Understanding normal and impaired word reading: Computational principles in quasi-regular domains. Psychological Review, 103, pp.56-115.

Rastle, K., \& Coltheart, M., 1999. Lexical and nonlexical phonological priming in reading aloud. Journal of Experimental Psychology, 25, pp.461-481.

Seidenberg, M. S., \& McClelland, J. L., 1989. A distributed, developmental model of word recognition and naming. Psychological Review, 96, pp.523-568.

Seidenberg, M. S., Petersen, A., MacDonald, M. C., \& Plaut, D. C., 1996. Pseudohomophe effects and models of word recognition. Journal of Experimental psychology, 22, pp.48-62.

Stone, G., \& Van Orden, G. C., 1993. Strategic control of processing in word recognition. Journal of Experimental Psychology: Human Perception and Performance, 19, pp.744-774.

Woodcock, R. W., 1998. Woodcock reading mastery tests-NU. Circle Pines, MN: American Guidance Service.

Ziegler, J. C., \& Goswami, 2005. Reading acquisition, developmental dyslexia, and skilled reading across languages: A psycholinguistic grain size theory. Psychological Bulletin, 131, pp.3-29. 